\section{Perioperative Management of a Patient with Large Anterior Mediastinal Mass and Cardiopulmonary Compromise: An Updated Algorithm}

Dean Flaten, M.D., Mahalia Marcotte, M.D., James Walker, M.D.

University of Kansas School of Medicine-Wichita, Department of Anesthesiology, Wichita, KS

Received August 9, 2019; Accepted for publication May 28, 2020; Published online July 10, 2020

\section{INTRODUCTION}

Patients who present with an anterior mediastinal mass (AMM) pose complex risks for the anesthesiologist to consider. While AMMs are quite rare, especially in the adult patient population, they are related most commonly to lymphoma, thymoma, germ cell tumor, granuloma, bronchogenic carcinoma, thyroid tumors, bronchogenic cyst, and cystic hygromas, in order of frequency.,

Since the 1970s, intraoperative complication rates of $7-20 \%$ have been reported, ranging from mild hypoxia and hypotension to complete cardiovascular collapse, airway compression, and death., ${ }^{3,4}$ When the best practices for anesthetic management of AMM's are adhered to, including maintenance of spontaneous ventilation and avoidance of supine positioning, mortality remains relatively low, ranging from $0.3-1.1 \% .^{5}$ However, some of these complications may be unavoidable despite adhering to standard practices for perioperative AMM management and require appropriate preparation and time-critical intervention to avoid serious injury or death. ${ }^{4}$

We present a brief overview of the important principles and practices concerning the management of AMM and suggest an updated algorithm with emphasis on preoperative risk stratification. Furthermore, we describe a unique case of significant hypoxemia with normotension in the setting of severe contralateral tracheobronchial and pulmonary artery compression to illustrate these points.

\section{CASE REPORT}

A 32-year-old male with history of hypertension and recently diagnosed massive AMM presented to a tertiary care facility to undergo parasternal mediastinotomy and anterior mediastinal biopsy. Written health care proxy consent was obtained.

Four months prior, the patient began experiencing symptoms consistent with gastroesophageal reflux disease (GERD) and was started on a proton pump inhibitor. The patient continued to experience GERD-like symptoms and positional chest pain with dyspnea. The patient was treated for an upper respiratory infection and given a short course of corticosteroids for presumed acute pericarditis; however, the patient continued to have evolution of his symptoms over the following months, including 15-pound unintentional weight loss and progressive nocturnal orthopnea with supine positioning.

He presented to an outlying facility for evaluation of acute left arm swelling. As part of that work-up, the patient underwent a chest CT with and without contrast, which revealed a heterogenous 14.5 $\mathrm{cm}$ in transverse diameter $\mathrm{x} 10.7 \mathrm{~cm}$ in antero-posterior diameter $\mathrm{x}$ $10.5 \mathrm{~cm}$ in cranio-caudad diameter mass within the antero-superior mediastinum. The mass demonstrated significant encasement and displacement of the great vessels. The left mainstem bronchus was 80 - 90\% compressed and the right and left pulmonary arteries were $80-90 \%$ and $40 \%$ compressed, respectively. The right mainstem bronchus, aorta, and superior vena cava appeared minimally, if at all, compressed (Figure 1). Subsequently, cardiothoracic surgery and oncology were consulted, and the patient was scheduled for biopsy of the mass via a left-sided anterior thoracotomy.

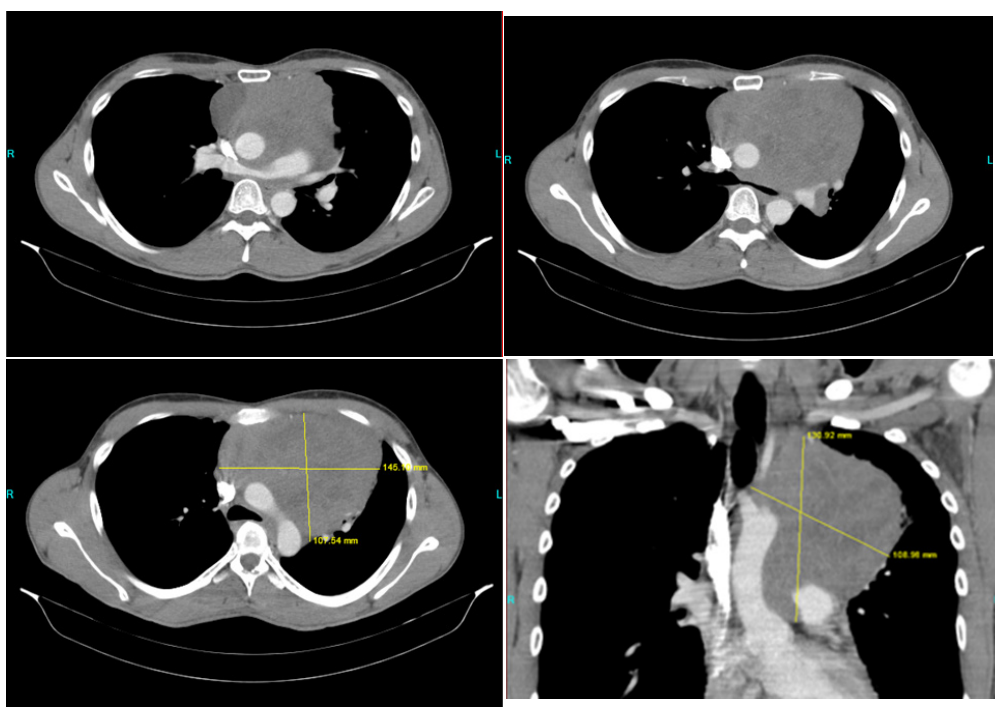

Figure 1. Chest CT with contrast. Top left: Axial image depicting severe left mainstem bronchus compression. Top right: Axial CT showing size of mass and mild-moderate supracarinal tracheal compression. Bottom left: Axial image showing severe right pulmonary artery compression. Bottom right: Coronal image demonstrating size of the AMM and special relationship to great vessels and aortic arch.

One week later, on the day of surgery, the patient was evaluated by the anesthesia team. Chest CT imaging and prior laboratory studies were reviewed. During the physical evaluation, the patient reported significant orthopnea and dyspnea with positioning despite saturating in the mid 90s on room air when in the upright or forward flexed position. The collaborative anesthetic and surgical plans were discussed, including preoperative arterial catheter placement, intraoperative flexible bronchoscopy, availability of armored and double lumen tubes, preparation for sternotomy, maintenance of spontaneous ventilation, and intraoperative ability to reposition to lateral, prone and sitting positions.

The patient was premedicated with $1 \mathrm{mg}$ intravenous (IV) midazolam. Intraoperatively, a combined induction with sevoflurane, $50 \mathrm{mg}$ IV ketamine, and $0.2 \mathrm{mg}$ IV glycopyrrolate was completed and laryngotracheal topical anesthesia spray with $4 \%$ lidocaine was applied to the glottis. A $7.0 \mathrm{~mm}$ armored endotracheal tube was passed into the trachea with a Grade 1 view. Spontaneous ventilation was maintained throughout. Dissection into the anterior mediastinum via left-sided parasternal mediastinotomy ensued. Upon surgical entry into the mediastinum, the patient developed significant hypoxia with oxygen saturations of $70-75 \%$, accompanied by a drop-in endtidal carbon dioxide from 37 to $20 \mathrm{mmHg}$ despite preservation of adequate expired tidal volumes, $100 \% \mathrm{FiO} 2$, and supportive manual 
KANSAS JOURNAL of MEDICINE

PERIOPERATIVE MANAGEMENT ANTERIOR

MEDIASTINAL MASS

continued.

adequate expired tidal volumes, $100 \% \mathrm{FiO} 2$, and supportive manual ventilation.

Flexible bronchoscopy was completed and the patient was noted to have moderate supra-carinal and severe left mainstem compression. Interventions made to improve oxygenation included repositioning the patient in left/right lateral, sitting, and prone positions. Improvement in both oxygenation and end-tidal carbon dioxide was achieved after closure of the mediastinotomy and turning the patient in the prone position. Based on the timing of improvement in oxygenation and normalization of end-tidal carbon dioxide, it was believed that the patient's hypoxemia was due primarily to left pulmonary artery compression after exposure to atmospheric pressure when the mediastinotomy incision was made. Despite the severe hypoxemia, the patient remained hemodynamically stable throughout the procedure via maintenance of cardiac output likely through an undiagnosed patent foramen ovale (PFO).

Postoperatively, the patient was sedated and transferred directly to the ICU in the prone position. Due to the patient bucking on the endotracheal tube and concern for harm to self, he was paralyzed with vecuronium. The patient was extubated inadvertently on postoperative day two while in the prone position. He subsequently went into cardiopulmonary arrest and expired.

\section{DISCUSSION}

Patients with large AMMs are at an increased risk for perioperative complications and death, even for non-mediastinal related surgery. ${ }^{3}$ As such, these patients demand prudent preoperative evaluation and preparation prior to surgery. Bechard et al. ${ }^{4}$ found that patients who presented with signs and symptoms of cardiopulmonary compromise (orthopnea, stridor, cyanosis, jugular venous distension, and superior vena cava syndrome), combined obstructive and restrictive pattern on pulmonary function tests, pertinent CT scan findings ( $>50 \%$ tracheal compression or pericardial effusion) and mass volumes greater than $130 \mathrm{~cm}$ are at increased risk for perioperative compromise., Our patient demonstrated several of the aforementioned signs and symptoms including airway compression, orthopnea, pericardial effusion and large mass volume, indicating his elevated perioperative risk.

The current standard for perioperative management of patients with AMM was developed in 1984 by Neuman et al. ${ }^{6}$ based upon three case reports. Their algorithm remains a standard in anesthesia textbooks. We propose an updated algorithm based on the most conservative approach, latest research, and advancements in CT, MRI, and ultrasound imaging (Figure 2).

The anesthesiologist should be cognizant of the inherent risks of anesthesia for patients with AMM, despite the relatively low mortality in adult patients.

The first priority is to avoid general anesthesia unless absolutely necessary prior to treatment of the mass. Multiple mechanisms for worsening airway and great vessel compression with general anesthesia have been implicated. Anesthesia induction decreases chest wall and airway tone and increases the transthoracic pressure with worsening extrinsic compression and lower lung volumes. ${ }^{7}$ This pathophysiology can be worsened with positive pressure ventilation. For this reason, all patients who can undergo CT-guided biopsy of their mass should do so prior to being subjected to general anesthesia. CT-guided AMM biopsies are reported to have a diagnostic accuracy of more than $90 \%$ and can be accomplished under local anesthesia with minimal sedation. ${ }^{1,8}$ If CT-guided biopsy and medical therapeutic interventions (chemotherapy, radiation, corticosteroids) cannot be completed, then most mediastinal biopsies may be accomplished using anterior mediastinoscopy or parasternal mediastinotomy under local anesthesia and sedation with dexmedetomidine and/or ketamine infusions. ${ }^{9}$

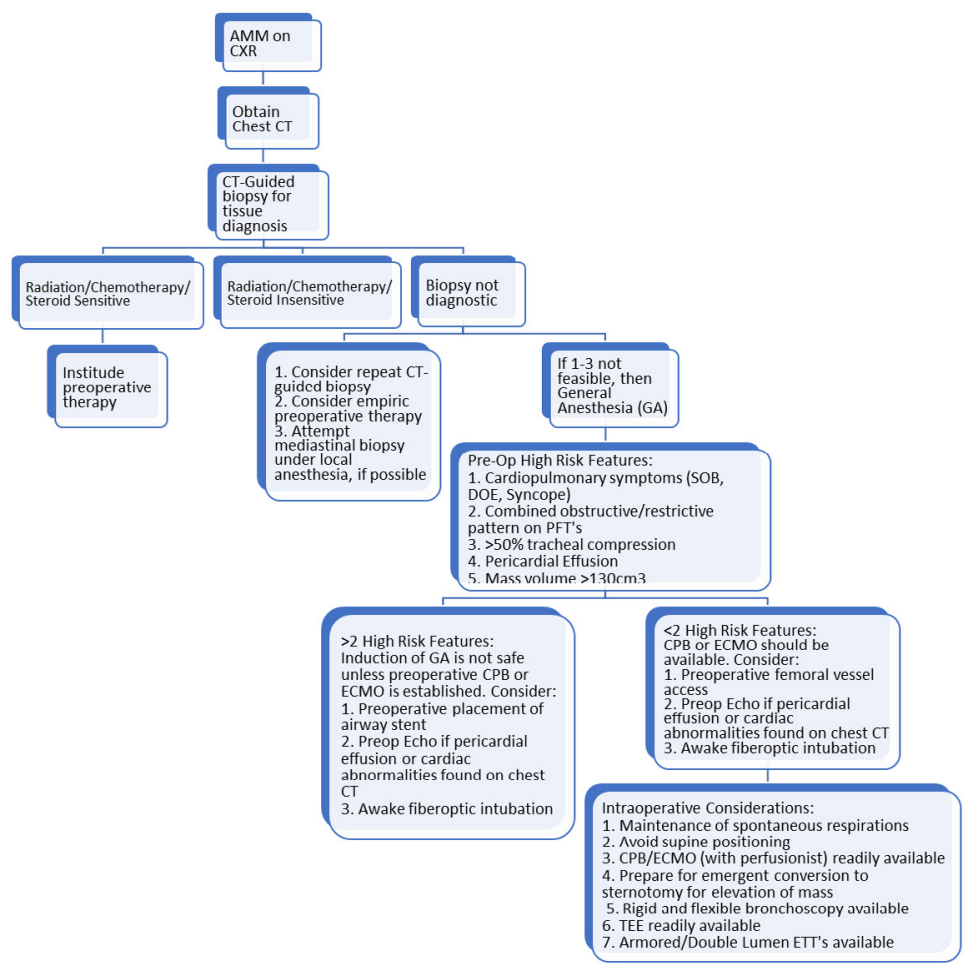

Figure 2. Proposed updated algorithm.

Previous algorithms have recommended routine utilization of upright and supine spirometry as part of the AMM preoperative evaluation. Recently, however, this recommendation has been challenged. ${ }^{10}$ Hnaituk et al. ${ }^{11}$ found that this recommendation, based largely on anecdotal data, was neither predictive of identifying patients at increased risk for perioperative complications, nor did it alter the choice of anesthetic technique compared to that with $\mathrm{CT}$ scan and symptomatology alone., ${ }^{411}$

In summary, the anesthetic management of patients with AMM can be difficult. Despite the low mortality rate and relatively low complication rate, these patients can experience severe and rapid decompensation with tragic outcomes if appropriate preanesthetic work-up and diligent preparations are not made. Furthermore, the importance of standby cardiopulmonary bypass (+/- pre-induction femoral arteriovenous access) cannot be underestimated, given that the time to initiate bypass becomes critically necessary if severe cardiopulmonary compromise occurs.,6,7,10 Other life-saving treatment modalities, which cannot be overlooked, include the 
ability to transition to sternotomy with subsequent elevation of the mass, change patient position, implement rigid bronchoscopy, and utilize intraoperative transesophageal echocardiography. ${ }^{3,4}$

Our patient illustrated a unique presentation and intraoperative challenge of a relatively rare disease process, in that he remained essentially asymptomatic until the mediastinal cavity was opened to atmospheric pressure. This led to decreased intrathoracic pressure and increased transthoracic pressure resulting in tracheobronchial and pulmonary artery compression causing $\mathrm{V} / \mathrm{Q}$ mismatch and near complete arteriovenous shunting, possibly via undiagnosed PFO. The latter findings presumably were due to severe bilateral $\mathrm{V} / \mathrm{Q}$ mismatch, increased right-sided dead space, and increased right-sided shunting. We are aware of only one other similar presentation in the literature as reported by Takeda et al. ${ }^{7}$ in 1999.

\section{REFERENCES}

${ }^{1}$ Slinger P, Karsli C. Management of the patient with a large anterior mediastinal mass: Recurring myths. Curr Opin Anesthesiol 2007; 20(1):1-3. PMID: 17211158.

${ }^{2}$ Sulen N, Petani B, Bacić I, Morović D. Anesthetic management of a patient with central airway compression due to posterior mediastinal mass. Acta Clin Croat 2016; 55(Suppl 1):103-107. PMID: 27276782.

${ }^{3}$ Gothard JW. Anesthetic considerations for patients with anterior mediastinal masses. Anesthesiol Clin 2008; 26(2):305-314. PMID: 18456215.

4 Béchard P, Létourneau L, Lacasse Y, Côté D, Bussiéres J. Perioperative cardiorespiratory complications in adults with mediastinal mass: Incidence and risk factors. Anesthesiology 2004; 100(4):826-834. PMID: 15087617.

${ }^{5}$ Gardner JC, Royster RL. Airway collapse with an anterior mediastinal mass despite spontaneous ventilation in an adult. Anesth Analg 2011; 113(2):239-242. PMID: 21596865.

${ }^{6}$ Neuman GG, Weingarten AE, Abramowitz RM, Kushins LG, Abramson AL, Ladner W. The anesthetic management of a patient with an anterior mediastinal mass. Anesthesiology 1984; 60(2):144-147. PMID: 6546480.

7 Takeda S, Miyoshi S, Omori K, Okumura M, Matsuda H. Surgical rescue for life-threatening hypoxemia caused by a mediastinal tumor. Ann Thorac Surg 1999; 68(6):2324-2326. PMID: 10617025.

${ }^{8}$ Chait P, Rico L, Amaral J, et al. Ultrasound guided core biopsy of mediastinal masses in children. Pediatr Radiol 2005; 35:S76.

9 Nafiu OO, Srinivasan A, Ravanbakht J, Wu B, Lau WC. Dexmedetomidine sedation in a patient with superior vena cava syndrome and extreme needle phobia. J Cardiothorac Vasc Anesth 2008; 22(4):581-583. PMID: 18662636.

${ }^{10}$ Rath L, Gullahorn G, Connolly N, Pratt T, Boswell G, Cornelissen C. Anterior mediastinal mass biopsy and resection: Anesthetic techniques and perioperative concerns. Semin Cardiothorac Vasc Anesth 2012; 16(4):235242. PMID: 22891051.

${ }^{11}$ Hnatiuk OW, Corcoran PC, Sierra A. Spirometry in surgery for anterior mediastinal masses. Chest 2001; 120(4):1152-1156. PMID: 11591553.

Keywords: mediastinal neoplasm, lymphoma, cardiopulmonary bypass, perioperative care, mediastinal disease 